\title{
Intraoperative Neurophysiological Monitoring in Spine Surgery
}

\author{
Mushtaq A Shaikh
}

\begin{abstract}
Intraoperative neurophysiological monitoring (IONM) encompasses a variety of neurological testing modalities used during surgery to monitor, identify, and potentially prevent iatrogenic complications to the nervous system and the blood supply to it or adjacent tissue. It has been used to monitor integrity of the neural pathway during spinal, neurological, cranial, and vascular procedures. Specific tests are designed to the clinical need of the surgery. Modalities may include: Somatosensory evoked potentials, motor evoked potentials, spontaneous or triggered electromyography, brainstem auditory evoked potentials, visual evoked potentials, electroencephalogram, and electrocorticography. During spinal surgery, a wide variety of procedures are utilized in which the spinal cord, nerve roots, and key blood vessels are frequently placed at risk from iatrogenic injury. Multimodal IONM relies on the strengths of different types of neurophysiological modalities to maximize the diagnostic efficacy in regard to sensitivity and specificity in the detection of impending neural injury. Thorough knowledge of the benefits and limitations of each modality helps in optimizing the diagnostic value of IONM during spinal procedures. As spinal surgeries continue to evolve, the value of neuromonitoring will also continue to become more prominent.
\end{abstract}

Keywords: Electroencephalography, Electromyography, Motor evoked potentials, Sensory evoked potentials.

How to cite this article: Shaikh MA. Intraoperative Neurophysiological Monitoring in Spine Surgery. J Postgrad Med Edu Res 2017;51(2):89-94.

Source of support: Nil

Conflict of interest: None

\section{INTRODUCTION}

Intraoperative neurophysiological monitoring (IONM) is a continually evolving field and ultimately, the aim is to preserve the integrity of neural structure. During spinal surgery, several structures are placed at risk for potential injury, including the spinal cord, nerve roots, lumbar plexus, and all relevant vascular supply to these structures. Several neurophysiological modalities are available today for monitoring various features of the

\footnotetext{
Director

Department of Intraoperative Neurophysiology, Bespoke Healthcare Ltd, Preston, UK

Corresponding Author: Mushtaq A Shaikh, Director, Department of Intraoperative Neurophysiology, Bespoke Healthcare Ltd Preston, UK, Phone: +441772700629, e-mail: mushtaq@ bespokehealthcare.co.uk
}

central and peripheral nervous system, each contributing to its own set of criterion as diagnostic techniques, including benefits, limitations, and sensitivity/specificity. The most frequently used modalities for spinal procedures are somatosensory evoked potentials (SSEPs), motor evoked potentials (MEPs), and spontaneous electromyography (sEMG) or triggered EMG (tEMG). To optimally preserve the neural structures during spinal surgery, an interdisciplinary communication among the surgical, neuromonitoring, and anesthetic team is imperative.

\section{SOMATOSENSORY EVOKED POTENTIAL}

The SSEP monitors the dorsal column-medial lemniscus pathway, which mediates tactile discrimination, vibration sensation, form recognition, and joint/muscle sensation (conscious proprioception). ${ }^{1}$ Skin, tendons, and muscle receptors generate information to these primary sensory modalities and relay signals to neurons whose soma are situated in dorsal root ganglia at all spinal levels, though SSEPs do not involve the spinothalamic (temperature and pain) pathway. Axons from these first-order neurons project to the spinal cord via the medial root entry zone, giving rise to the fasciculi gracilis and cuneatus, which subsequently carry sensory information from the lower and upper extremities respectively. The first synapse in the sensory pathway occurs in the lower medulla. These tracts then ascend along the dorsal columns in the spinal cord. After decussation, the medial lemniscus is formed at the medullary level; it ascends to the thalamus and finally transmits sensory information to the primary somatosensory cortex.

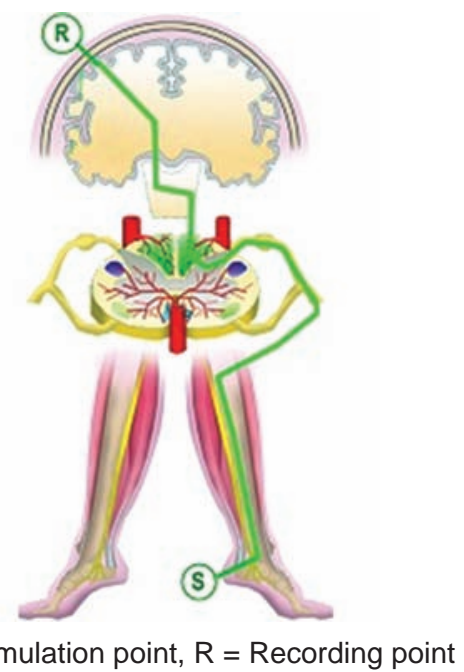

$\mathrm{S}=$ Stimulation point, $\mathrm{R}=$ Recording point 


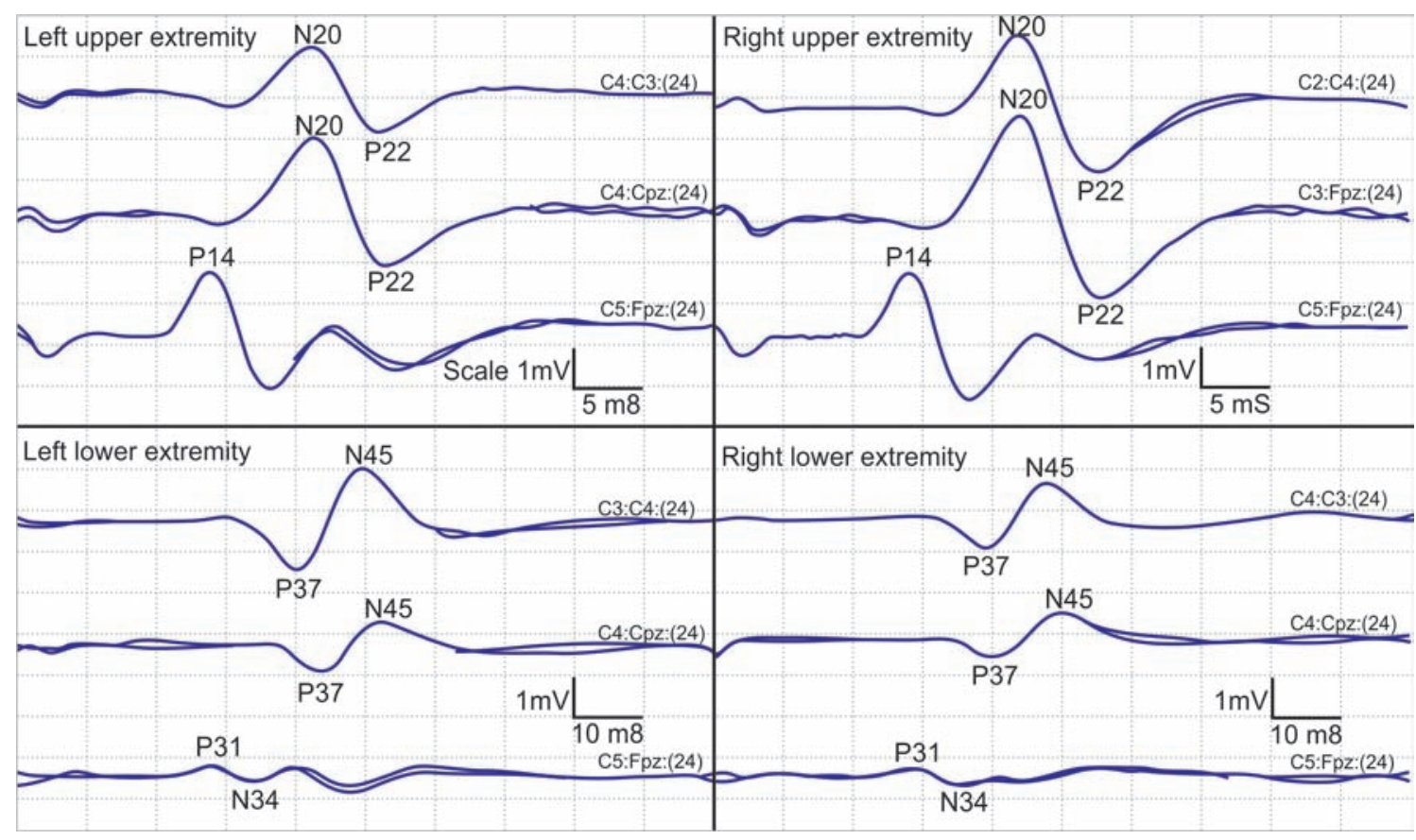

Fig. 1: Upper and lower limb SSEP responses. The negative potential measured corresponding to the upper extremities at $20 \mathrm{~ms}$ is known as the N20 response, and a positive potential measured corresponding to the lower extremities at $37 \mathrm{~ms}$ is known as the $\mathrm{P} 37$ response

The SSEPs involve electrical stimulation of mixed sensory and motor fibers caudal to the region of the spinal cord at risk, paired with recording of these signals rostral to the region at risk (typically at the dorsal neck and scalp). Electrical stimulation of the peripheral nerves produces major positive and negative part of the waveform in the somatosensory cortex (Fig.1).

Alarm criteria of a $50 \%$ reduction in amplitude and/or a $10 \%$ increase in latency are generally used as guidelines for notifying the surgeon of a potential deficit, and corrective intervention should be considered if these changes correspond to a particular surgical manipulation. The neuromonitoring team must be well aware of the external interferences, particularly caused by surrounding electrical equipments, and artifacts to reduce false-positive interpretations. Factors that affect the SSEP amplitude include volatile gaseous agents, hypothermia, hypotension, nitrous oxide, and electrical interference. The most common factor that affects SSEP latency readings is temperature. Any SSEP changes with amplitude reduction of more than $50 \%$ should also be considered relevant if they are temporally associated with a specific surgical intervention, such as during placement of spinal instrumentation or during correction of a spinal deformity. Nuwer et $\mathrm{al}^{2}$ appraised the efficacy of monitoring intraoperative SSEP during scoliosis surgery in 1995 in a large multicenter survey of over 50,000 spinal surgeries. They concluded that the ability of SSEPs to detect new postoperative neu- rological deficits has an overall sensitivity of $92 \%$ and specificity of $98.9 \%$.

\section{MOTOR EVOKED POTENTIALS}

In the last 30 years, MEPs have become extremely valuable in IONM. In 1980, Merton and Morton ${ }^{3}$ demonstrated that a high-voltage pulse applied transcranially could elicit contralateral motor response. The single-pulse stimulation technique was found to be highly sensitive to anesthetic effects, and the technique has since been revised to using multipulse stimulation along with various modifications in anesthetic regimens to obtain maximize motor response. By the early 1990s, transcranial electrical stimulation was popularized as a method to monitor the corticospinal tracts ${ }^{4}$ (Fig. 2).

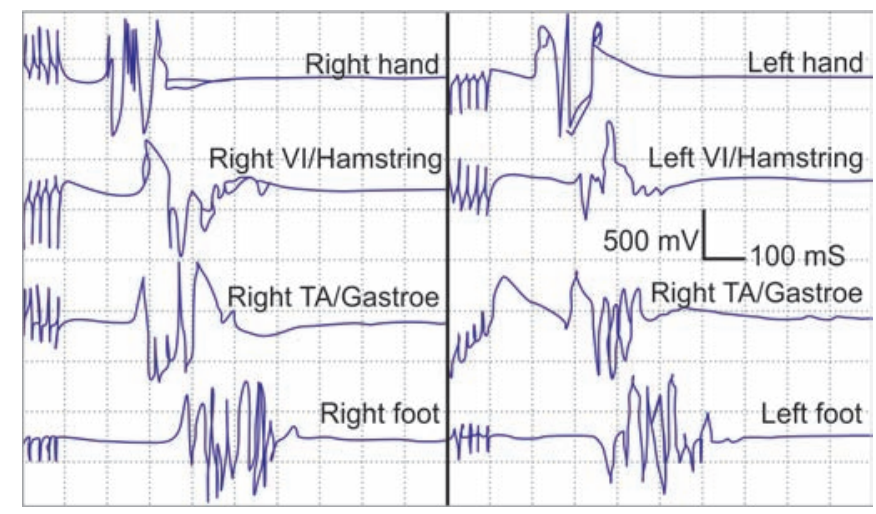

Fig. 2: Normal illustration of MEPs from intrinsic hand muscles, vastus lateralis, hamstrings, tibialis anterior, gastrocnemius (Gastroc), and plantar foot muscles 
Prior to the widespread use of MEP monitoring, the only way to assess corticospinal tract integrity and resulting motor function during surgery was the Stagnara wake-up test, which involved waking patients during surgery and asking them to move their feet. ${ }^{5,6}$ This test lacks the obvious benefit of continuous assessment of neural function, and its use is limited in certain patients, such as those with cognitive or hearing deficits. If, however, the test is positive and the patient has sustained a motor deficit, it is possible that a substantial period of time has elapsed between the injury, its detection, and the ultimate intervention.

As MEPs effectively monitor function of the corticospinal tract (a pathway, i.e., not covered by SSEP monitoring), changes in MEPs are more sensitive in the detection of postoperative motor deficits. ${ }^{7,8}$ There are many reported cases of patients with motor deficits following surgery in which when SSEPs alone were utilized they had failed to demonstrate any alarm criteria. ${ }^{9}$ The deficits in these cases were thought to have been caused either through direct mechanical trauma or vascular event of the corticospinal tracts. The dorsal columns and the corticospinal tract appear in different vascular and anatomical territories, as the dorsal columns receives the perfusion mainly via the posterior spinal arteries, whereas the lateral corticospinal tracts, the anterior corticospinal tracts, and the anterior horn cells receive the majority of their blood supply via the anterior spinal artery. During scoliosis surgery, for example, small radiculomedullary arteries passing between adjacent vertebrae may be stretched or compressed during correctional maneuvers, resulting in ischemia or infarction; then postoperative motor deficits may occur without changes in SSEPs - with changes detected only in MEP monitoring, if such an injury affected only the anterolateral funiculus.

The MEPs can be stimulated either transcranially or via direct cortical stimulation. Transcranial stimulation is currently performed electrically. For standard transcranial MEP recording (TCMEP), stimulation electrodes are placed at C3 and C4 (10-20 International System) for activation of both upper and lower extremity muscle groups, with alternative sites at $\mathrm{C} 1$ and $\mathrm{C} 2$ if more focal activation of the lower extremity muscle groups is desired. The stimulation intensity alters the current field size and distribution to the cortex and subcortical fibers. Subcortical white matter motor tracts are activated at the axon exiting the grey matter, or entering the internal capsule or even brainstem, which is not an issue when the structures at risk are located below the foramen magnum, as is the case in spine surgery.

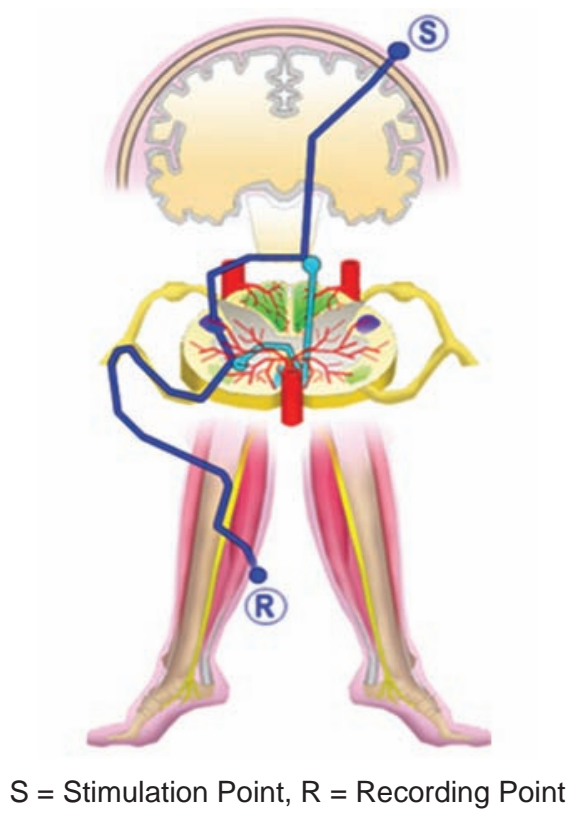

\section{Interpretation of MEP Recording}

At present, four different criteria are utilized for interpreting TcMEP responses:

1. The "all-or-nothing"

2. The amplitude

3. The morphology

4. The threshold

The "all-or-nothing" is the most predominantly used method. Therefore, a complete absence of the MEP response from an initial baseline recording is indicative of a clinically significant event. Because of the all-or-nothing nature of this interpretation, it has been proposed that this method is not sensitive enough in detecting subtle deficits involving the corticospinal tract that may still result in postoperative motor deficits and are potentially correctable if detected. ${ }^{8}$

A variation of the "all-or-nothing" criterion involves measuring the initial baseline compound muscle action potential (CMAP) amplitude, thus any relative changes in amplitude from the baseline indicates if a clinically significant change has occurred. The amplitude criterion, as described by Langeloo et al, ${ }^{10}$ uses an $80 \%$ amplitude decrement in at least one out of six recording sites as a criterion for a clinically significant change. This criterion used in a study of 142 patients achieved a sensitivity of $100 \%$ and a specificity of $91 \%$.

A similar form of reasoning can be applied to the threshold criterion, which analyses the increase in stimulation threshold required to maintain CMAP responses. Calancie and Molano ${ }^{9}$ reported that increases in the threshold of $100 \mathrm{~V}$ or more required for eliciting CMAP responses that are persistent for 1 or more hours and not due to systemic factors were highly correlated with postoperative motor deficits. 
The morphology criterion accounts for reduced motor conduction of the corticospinal tracts by looking at changes in the duration and the morphology of MEP waveforms. Quinones-Hinojosa et $\mathrm{al}^{11}$ observed changes in the CMAP waveform elicited during motor stimulation, from a polyphasic to biphasic waveform or from polyphasic to biphasic and ultimately to loss of signals, correlating significantly with postoperative motor deficits.

\section{Anesthetic Consideration for IONM}

For optimal acquisition of signals, total IV anesthesia (TIVA) is used, in which compounds, such as nitrous oxide, volatile agents, and muscle relaxants are excluded, and short-acting agents, such as fentanyl and propofol are relied upon to achieve anesthetic control. Although this may pose more of a challenge to the anesthesia team and possibly the surgeon, given potential movement of the patient, TIVA offers clear benefits in obtaining MEPs over inhaled anesthetics. ${ }^{12}$ Anesthetic inhalants decrease the possible pool of motoneurons available for recruiting. Since the propagation of a peripheral motor response is dependent on indirect wave (I-wave) generation, inhaled agents may interfere with MEP acquisition as they inhibit the interneuron generators of I-waves at the level of the cerebral cortex and the anterior horn cells. Higher levels of propofol used in TIVA will likely causes attenuation of alpha-motoneurons; a factor that requires consideration when interpreting MEP loss or amplitude reduction in this setting. ${ }^{13}$ There are of course contraindications with TcMEP monitoring, such as with patients with deep brain stimulators or cochlear implants. Such contraindications to MEP monitoring has to be weighed up against the benefits provided by the monitoring. However, despite TcMEP being relatively safe, tongue laceration (the most common complication) can occur due to contraction of strong facial muscles. ${ }^{14} \mathrm{~A}$ bite block is essential to avoid this complication.

D-waves is another technique of recording MEPs signals directly from the spinal cord. This recording technique requires the placement of a direct epidural recording electrode and is thus typically limited to recording above the level of T11. The major benefits of this modality have been reported during intramedullary spinal cord tumor resection. ${ }^{15,16}$ Direct corticospinal waves have been shown to be closely correlated with the postoperative clinical status, in which a complete loss of MEPs with at least $50 \%$ preservation of the D-wave amplitude generally results in a transient paraplegia. Furthermore, patients with the loss of D-wave amplitude completely during surgery may conceivably suffer permanent motor deficits. ${ }^{17}$

\section{SPONTANEOUS EMG}

Spontaneous EMG activity is used during spinal surgery to monitor the corresponding nerve roots responsible for muscle innervation. Any surgical manipulation, such as pulling, stretching, or compression of nerves produces neurotonic discharges resulting in activity in the corresponding innervated muscle(s). For example, during cervical spine procedures, the $\mathrm{C} 5$ nerve root is at particular risk of injury and requires particular attention in monitoring. For this reason, concurrent monitoring of two muscles is recommended to minimize the risk of C5 nerve root injury. The deltoid (predominantly C5, also C6) and biceps brachii (predominantly C6, also C5) muscles are typically used to monitor the C5 level. Spontaneous EMG is highly sensitive to irritation of the nerve root due to manipulation, retraction, and irrigation during surgery.

Spontaneous EMG activity are described as spikes, bursts, or trains. During surgery, "trains" are of clinical significance and the surgeon must be notified of these activities (Fig. 3). Repetitive EMG firing caused by continuous force applied to the nerve root produces continuous "train" activity. Trains of higher frequency and / or amplitude tend to represent significant nerve fiber recruitment caused by excessive force on the nerve and are likely to indicate a high probability of nerve injury if a relevant manipulation is sustained. Spontaneous EMG spikes and bursts, on the contrary, often can indicate proximity to the nerve root. Occasionally interferences will be picked up from various sources, such as electrocautery devices, high-speed drills, and other surrounding electrical devices that may be mistaken for spiking or

Left tibialis anterior

Fig. 3: Note the sEMG activity in the left tibialis anterior muscle 
trains of EMG activity. Anesthetic requirements for sEMG mandate that no paralytic agents are used, and that trainof-four testing should indicate that at least three out of four be present for sEMG to be of value. It is essential to know the patient's any neurological disorders that may interfere with the ability to acquire EMG activities. Condition, such as myasthenia gravis, botulinum toxin used to treat dystonia and muscular dystrophy are classic examples of neurological conditions that interfere with EMG activities.

\section{TRIGGERED EMG (Pedicle Screw Stimulation)}

Over the past two decades, segmental instrumentation and fusion using pedicle screw constructs have become the standard for spinal stabilization. A potential risk of pedicle screw placement is a screw breach of the medial pedicle wall into the spinal canal. Triggered EMG is a technique used to determine screw breach of the medial or inferior pedicle wall. An accurately placed pedicle screw surrounded by the bone acts as an insulator to electrical conduction. Higher level of electrical current is therefore, required to stimulate the surrounding nerve root. Typically, a monopolar electrode is used to directly stimulate the top of the pedicle screw at increasing current intensities.

When a medial pedicle wall breached, the threshold of stimulation intensity is significantly reduced. Therefore, different stimulation thresholds exist due to the variation in thickness and shape between thoracic and lumbar pedicles. Previous studies have determined that a threshold less than $7 \mathrm{~mA}$ for a probe stimulation, or $10 \mathrm{~mA}$ for a screw stimulation, advocates a medial wall breach in the lumbar pedicles. ${ }^{18} \mathrm{~A}$ threshold reading between 10 and $15 \mathrm{~mA}$ indicates a credible likelihood that no of breach of the medial wall has occurred, whereas thresholds $>15$ mA signify a $98 \%$ assurance of accurate screw positioning, as seen on postoperative computed tomography scan. ${ }^{19}$ Threshold values above $20 \mathrm{~mA}$ assure with great certainty that there is no medial pedicle wall breach. Stimulation thresholds $<6 \mathrm{~mA}$ for thoracic pedicle screw placement suggest a medial pedicle breach. ${ }^{20}$

False-negative responses can occur during pedicle screw stimulation as a result of various factors. This includes the use of muscles relaxants, preexisting nerve damage, or current spread; particularly, if the stimulation intensity is too high. It is important therefore, to take these factors into consideration to guarantee the precision of testing. The strength of muscle relaxation can be measured using a "train-of-four" test. Just as in the case of sEMG monitoring, tEMG monitoring requires that no paralytic agents be used and that four out of four twitches are optimal for reliable recording. In particular, the attention should to be directed to blood, fluid, or soft tissue around the head of the screw when stimulating. This could cause "shorting" and potentially shunt current away from the screw. It is worth mentioning that the hydroxyapatite coating on pedicle screws reduces the conductive capacity of the screws and thereby reduces the capability of electrical stimulation to detect a breach.

\section{MULTIMODAL IONM}

Multimodal monitoring has the potential to compensate for limitations of each individual monitoring modality and has become standard practice for a variety of spinal procedures. A combination of SSEP and MEP monitoring have been used in scoliosis surgery for combined monitoring of ascending and descending pathways. The inclusion of free-run EMG and tEMG/sEMG can enhance detection of nerve root injuries. Studies carried out previously have demonstrated sensitivities and specificities approximating 100\% for combined multimodal neuromonitoring. ${ }^{8,21}$

The concept of multimodal monitoring has become standard practice for a variety of spinal procedures. The individual strengths of each of its submodalities have been utilized in scoliosis surgery for combined monitoring of ascending and descending pathways, and thus, a more global and accurate assessment of the dorsal and ventral pathways of the spinal cord is achieved. With the inclusion of EMG monitoring, comprehensive function of the entire nervous system can be monitored, from cortex, spinal cord, nerve roots, peripheral nerves, and finally to the muscles. The combined use of SSEPs, MEPs both sEMG and tEMG provide the necessary tools required to optimally monitor the functional integrity of the spinal cord during varieties of routine and complex spinal surgeries, while maximizing the diagnostic efficacy of monitoring in detecting neurological injury. ${ }^{22-24}$ The virtually real-time information conveyed on the integrity of these monitoring parameters provided by IONM adds a stratum of security for the surgeons where neurological injury is an impending possibility during complex procedures. Figure 4 illustrates the utilization of multimodal IONM.

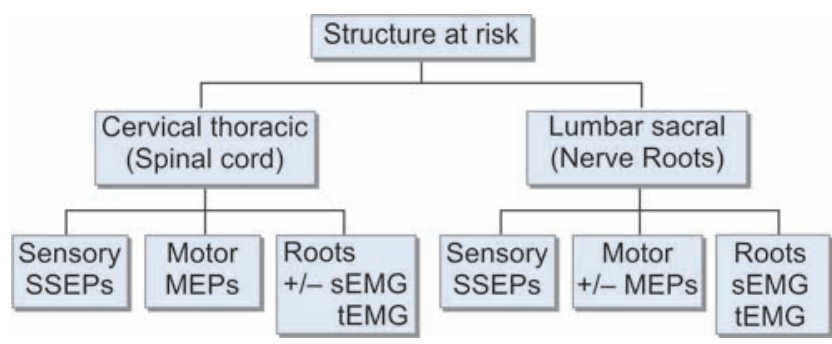

Fig. 4: Overview of frequently used IONM modalities for different spine regions 


\section{CONCLUSION}

Multimodality IONM is extremely valuable in the prevention of iatrogenic neurological injury during spine procedures. It is a dynamically evolving field with the potential to greatly improve the safety of spinal surgery. An appreciation of the benefits and limitations of each modality will help maximize the diagnostic value of IONM during spinal procedures. Preoperative interdisciplinary discussion between the neurosurgeon, anesthetist, and neurophysiologist is important in preventing neurological injury. Preoperative topics should include anesthetic requirements for IONM, alarm criteria to be used, and steps to be taken in response to a positive alarm.

\section{REFERENCES}

1. Chiappa, K.; Hill, R. Short latency somatosensory evoked potentials: methodology. In: Chiappa K, editor. Evoked potentials in clinical medicine. Philadelphia: Lippincott-Raven; 1997.

2. Nuwer MR, Dawson EG, Carlson LG, Kanim LE, Sherman JE. Somatosensory evoked potential spinal cord monitoring reduces neurologic deficits after scoliosis surgery: results of a large multicenter survey. Electroencephalogr Clin Neurophysiol 1995 Jan;96(1):6-11.

3. Merton PA, Morton HB. Stimulation of the cerebral cortex in the intact human subject. Nature 1980 May;285(5762):227.

4. Burke D, Hicks R, Stephen J, Woodforth I, Crawford M. Assessment of corticospinal and somatosensory conduction simultaneously during scoliosis surgery. Electroencephalogr Clin Neurophysiol 1992 Dec;85(6):388-396.

5. Ben-David B, Taylor PD, Haller GS. Posterior spinal fusion complicated by posterior column injury. A case report of a false-negative wake-up test. Spine (Phila Pa 1976) 1987 JulAug;12(6):540-543.

6. Vauzelle C, Stagnara P, Jouvinroux P. Functional monitoring of spinal cord activity during spinal surgery. Clin Orthop Relat Res 1973 Jun;93:173-178.

7. Hilibrand AS, Schwartz DM, Sethuraman V, Vaccaro AR, Albert TJ. Comparison of transcranial electric motor and somatosensory evoked potential monitoring during cervical spine surgery. J Bone Joint Surg Am 2004 Jun;86-A(6): 1248-1253.

8. Hsu B, Cree AK, Lagopoulos J, Cummine JL. Transcranial motor-evoked potentials combined with response recording through compound muscle action potential as the sole modality of spinal cord monitoring in spinal deformity surgery. Spine (Phila Pa 1976) 2008 May;33(10):1100-1106.

9. Calancie B, Molano MR. Alarm criteria for motor-evoked potentials: what's wrong with the "presence-or-absence" approach? Spine (Phila Pa 1976) 2008 Feb;33(4):406-414.

10. Langeloo DD, Lelivelt A, Louis Journee H, Slappendel R, de Kleuver M. Transcranial electrical motor-evoked potential monitoring during surgery for spinal deformity: a study of 145 patients. Spine (Phila Pa 1976) 2003 May;28(10):1043-1050.
11. Quinones-Hinojosa A, Lyon R, Zada G, Lamborn KR, Gupta N, Parsa AT, McDermott MW, Weinstein PR. Changes in transcranial motor evoked potentials during intramedullary spinal cord tumor resection correlate with postoperative motor function. Neurosurgery 2005 May;56(5):982-993.

12. Pechstein U, Nadstawek J, Zentner J, Schramm J. Isoflurane plus nitrous oxide versus propofol for recording of motor evoked potentials after high frequency repetitive electrical stimulation. Electroencephalogr Clin Neurophysiol 1998 Mar;108(2):175-181.

13. Kalkman CJ, Drummond JC, Kennelly NA, Patel PM, Partridge BL. Intraoperative monitoring of tibialis anterior muscle motor evoked responses to transcranial electrical stimulation during partial neuromuscular blockade. Anesth Analg 1992 Oct;75(4):584-589.

14. Macdonald DB. Intraoperative motor evoked potential monitoring: overview and update. J Clin Monit Comput 2006 Oct;20(5):347-377.

15. Deletis V, Sala F. Intraoperative neurophysiological monitoring of the spinal cord during spinal cord and spine surgery: a review focus on the corticospinal tracts. Clin Neurophysiol 2008 Feb;119(2):248-264.

16. Sala F. Improving spinal cord monitoring: a neurosurgeon's view. Clin Neurophysiol 2009 Feb;120(4):649-650.

17. Morota N, Deletis V, Constantini S, Kofler M, Cohen H, Epstein FJ. The role of motor evoked potentials during surgery for intramedullary spinal cord tumors. Neurosurgery 1997 Dec;41(6):1327-1336.

18. Calancie B, Madsen P, Lebwohl N. Stimulus-evoked EMG monitoring during transpedicular lumbosacral spine instrumentation. Initial clinical results. Spine (Phila Pa 1976) 1994 Dec;19(24):2780-2786.

19. Shi YB, Binette M, Martin WH, Pearson JM, Hart RA. Electrical stimulation for intraoperative evaluation of thoracic pedicle screw placement. Spine (Phila Pa 1976) 2003 Mar;28(6): 595-601.

20. Raynor BL, Lenke LG, Kim Y, Hanson DS, Wilson-Holden TJ, Bridwell KH, Bridwell KH, Padberg AM. Can triggered electromyograph thresholds predict safe thoracic pedicle screw placement? Spine (Phila Pa 1976) 2002 Sep;27(18):2030-2035.

21. Khealani B, Husain AM. Neurophysiologic intraoperative monitoring during surgery for tethered cord syndrome. J Clin Neurophysiol 2009 Apr;26(2):76-81.

22. Kelleher MO, Tan G, Sarjeant R, Fehlings MG. Predictive value of intraoperative neurophysiological monitoring during cervical spine surgery: a prospective analysis of 1055 consecutive patients. J Neurosurg Spine 2008 Mar;8(3):215-221.

23. MacDonald DB, Al Zayed Z, Khoudeir I, Stigsby B. Monitoring scoliosis surgery with combined multiple pulse transcranial electric motor and cortical somatosensory-evoked potentials from the lower and upper extremities. Spine (Phila Pa 1976) 2003 Jan;28(2):194-203.

24. QuraishiNA,LewisSJ,KelleherMO,SarjeantR, RampersaudYR, Fehlings MG. Intraoperative multimodality monitoring in adult spinal deformity: analysis of a prospective series of one hundred two cases with independent evaluation. Spine (Phila Pa 1976) 2009 Jun;34(14):1504-1512. 